\title{
Iterative Methods for Family of Strictly Pseudocontractive Mappings and System of Generalized Mixed Equilibrium Problems and Variational Inequality Problems
}

\author{
Yekini Shehu \\ Mathematics Institute, African University of Science and Technology, Abuja, Nigeria \\ Correspondence should be addressed to Yekini Shehu, deltanougt2006@yahoo.com \\ Received 6 September 2010; Accepted 25 November 2010 \\ Academic Editor: S. Al-Homidan \\ Copyright (C) 2011 Yekini Shehu. This is an open access article distributed under the Creative \\ Commons Attribution License, which permits unrestricted use, distribution, and reproduction in \\ any medium, provided the original work is properly cited. \\ We introduce a new iterative scheme by hybrid method for finding a common element of the set \\ of common fixed points of infinite family of $k$-strictly pseudocontractive mappings and the set of \\ common solutions to a system of generalized mixed equilibrium problems and the set of solutions \\ to a variational inequality problem in a real Hilbert space. We then prove strong convergence of \\ the scheme to a common element of the three above described sets. We give an application of our \\ results. Our results extend important recent results from the current literature.
}

\section{Introduction}

Let $K$ be a nonempty closed and convex subset of a real Hilbert space $H$. A mapping $A$ : $K \rightarrow H$ is called monotone if

$$
\langle A x-A y, x-y\rangle \geq 0, \quad \forall x, y \in K
$$

A mapping $A: K \rightarrow H$ is called inverse-strongly monotone (see, e.g., [1, 2]) if there exists a positive real number $\lambda$ such that $\langle A x-A y, x-y\rangle \geq \lambda\|A x-A y\|^{2}$, for all $x, y \in K$. For such a case, $A$ is called $\lambda$-inverse-strongly monotone. A $\lambda$-inverse-strongly monotone is sometime called $\lambda$-cocoercive. A mapping $A$ is said to be relaxed $\lambda$-cocoercive if there exists $\lambda>0$ such that

$$
\langle A x-A y, x-y\rangle \geq-\lambda\|A x-A y\|^{2}, \quad \forall x, y \in K .
$$


$A$ is said to be relaxed $(\lambda, \gamma)$-cocoercive if there exist $\lambda, \gamma>0$ such that

$$
\langle A x-A y, x-y\rangle \geq-\lambda\|A x-A y\|^{2}+\gamma\|x-y\|^{2}, \quad \forall x, y \in K .
$$

A mapping $A: H \rightarrow H$ is said to be $\mu$-Lipschitzian if there exists $\mu \geq 0$ such that

$$
\|A x-A y\| \leq \mu\|x-y\|, \quad x, y \in H .
$$

Let $A: K \rightarrow H$ be a nonlinear mapping. The variational inequality problem is to find an $x^{*} \in K$ such that

$$
\left\langle A x^{*}, y-x^{*}\right\rangle \geq 0, \quad \forall y \in K
$$

(See, e.g., $[3,4]$.$) We will denote the set of solutions of the variational inequality problem$ (1.5) by $\mathrm{VI}(K, A)$.

A monotone mapping $A$ is said to be maximal if the graph $G(A)$ is not properly contained in the graph of any other monotone map, where $G(A):=\{(x, y) \in H \times H$ : $y \in A x\}$ for a multivalued mapping $A$. It is also known that $A$ is maximal if and only if for $(x, f) \in H \times H,\langle x-y, f-g\rangle \geq 0$ for every $(y, g) \in G(A)$ implies $f \in A x$. Let $A$ be a monotone mapping defined from $K$ into $H$ and $N_{K} q$ a normal cone to $K$ at $q \in K$, that is, $N_{K} q=\{p \in H:\langle q-u, p\rangle \geq 0$, for all $u \in K\}$. Define a mapping $M$ by

$$
M q= \begin{cases}A q+N_{K} q, & q \in K, \\ \emptyset, & q \notin K .\end{cases}
$$

Then, $M$ is maximal monotone and $x^{*} \in M^{-1}(0) \Leftrightarrow x^{*} \in \mathrm{VI}(K, A)$ (see, e.g., [5]).

A mapping $T: K \rightarrow K$ is said to be $k$-strictly pseudocontractive if there exists a constant $k \in[0,1)$ such that

$$
\|T x-T y\|^{2} \leq\|x-y\|^{2}+k\|(I-T) x-(I-T) y\|^{2},
$$

for all $x, y \in K$. If $k=0$, then the mapping $T$ is nonexpansive. A point $x \in K$ is called a fixed point of $T$ if $T x=x$. The fixed points set of $T$ is the set $F(T):=\{x \in K: T x=x\}$. Iterative approximation of fixed points of $k$-strictly pseudocontractive mappings have been studied extensively by many authors (see, e.g., $[1,6-9]$ and the references contained therein).

Let $\varphi: K \rightarrow \mathbb{R}$ be a real-valued function and $A: K \rightarrow H$ a nonlinear mapping. Suppose $F: K \times K$ into $\mathbb{R}$ is an equilibrium bifunction. That is, $F(u, u)=0$, forall $u \in K$. The generalized mixed equilibrium problem is to find $x \in K$ (see, e.g., [10-12]) such that

$$
F(x, y)+\varphi(y)-\varphi(x)+\langle A x, y-x\rangle \geq 0,
$$

for all $y \in K$. We shall denote the set of solutions of this generalized mixed equilibrium problem by $\operatorname{GMEP}(F, A, \varphi)$. Thus,

$$
\operatorname{GMEP}(F, A, \varphi):=\left\{x^{*} \in K: F\left(x^{*}, y\right)+\varphi(y)-\varphi\left(x^{*}\right)+\left\langle A x^{*}, y-x^{*}\right\rangle \geq 0, \forall y \in K\right\} .
$$


If $\varphi=0, A=0$, then problem (1.8) reduces to equilibrium problem studied by many authors (see, e.g., $[8,13-17]$ ), which is to find $x^{*} \in K$ such that

$$
F\left(x^{*}, y\right) \geq 0
$$

for all $y \in K$. The set of solutions of (1.10) is denoted by $\operatorname{EP}(F)$.

If $\varphi=0$, then problem (1.8) reduces to generalized equilibrium problem studied by many authors (see, e.g., [18-20]), which is to find $x^{*} \in K$ such that

$$
F\left(x^{*}, y\right)+\left\langle A x^{*}, y-x^{*}\right\rangle \geq 0
$$

for all $y \in K$. The set of solutions of (1.11) is denoted by EP.

If $A=0$, then problem (1.8) reduces to mixed equilibrium problem considered by many authors (see, e.g., [21-23]), which is to find $x^{*} \in K$ such that

$$
F\left(x^{*}, y\right)+\varphi(y)-\varphi\left(x^{*}\right) \geq 0
$$

for all $y \in K$. The set of solutions of (1.12) is denoted by MEP.

The generalized mixed equilibrium problems include fixed-point problems, optimization problems, variational inequality problems, Nash equilibrium problems, and equilibrium problems as special cases (see, e.g., [24]). Numerous problems in Physics, optimization, and economics reduce to find a solution of problem (1.8). Several methods have been proposed to solve the fixed-point problems, variational inequality problems and equilibrium problems in the literature (see, e.g., $[5,11,12,20,25-30]$ ).

Recently, Ceng and Yao [25] introduced a new iterative scheme of approximating a common element of the set of solutions to mixed equilibrium problem and set of common fixed points of finite family of nonexpansive mappings in a real Hilbert space $H$. In their results, they imposed the following condition on a nonempty closed and convex subset $K$ of $H$ :

(E) $A: K \rightarrow \mathbb{R}$ is $\eta$-strongly convex and its derivative $A^{\prime}$ is sequentially continuous from weak topology to the strong topology.

We remark here that this condition (E) has been used by many authors for approximation of solution to mixed equilibrium problem in a real Hilbert space (see, e.g., $[31,32])$. However, it is observed that the condition (E) does not include the case $A(x)=\|x\|^{2} / 2$ and $\eta(x, y)=x-y$. Furthermore, Peng and Yao [21], R. Wangkeeree and R. Wangkeeree [30], and many other authors replaced condition (E) with the following conditions:

(B1) for each $x \in H$ and $r>0$, there exists a bounded subset $D_{x} \subseteq K$ and $y_{x} \in K$ such that for any $z \in K \backslash D_{x}$,

$$
F\left(z, y_{x}\right)+\varphi\left(y_{x}\right)-\varphi(z)+\frac{1}{r}\left\langle y_{x}-z, z-x\right\rangle<0
$$

or

(B2) $K$ is a bounded set. 
Consequently, conditions (B1) and (B2) have been used by many authors in approximating solution to generalized mixed equilibrium (mixed equilibrium) problems in a real Hilbert space (see, e.g., $[21,30]$ ). method.

Recently, Takahashi et al. [33] proved the following convergence theorem using hybrid

Theorem 1.1 (Takahashi et al. [33]). Let $K$ be a nonempty closed and convex subset of a real Hilbert space $H$. Let $T$ be a nonexpansive mapping of $K$ into itself such that $F(T) \neq \emptyset$. For $C_{1}=K, x_{1}=P_{C_{1}} x_{0}$, define sequences $\left\{x_{n}\right\}_{n=0}^{\infty}$ and $\left\{y_{n}\right\}_{n=1}^{\infty}$ of $K$ as follows:

$$
\begin{gathered}
y_{n}=\alpha_{n} x_{n}+\left(1-\alpha_{n}\right) T x_{n}, \quad n \geq 1, \\
C_{n+1}=\left\{z \in C_{n}:\left\|y_{n}-z\right\| \leq\left\|x_{n}-z\right\|\right\}, \quad n \geq 1, \\
x_{n+1}=P_{C_{n+1}} x_{0}, \quad n \geq 1 .
\end{gathered}
$$

Assume that $\left\{\alpha_{n}\right\}_{n=1}^{\infty} \subset[0,1)$ satisfies $0 \leq \alpha_{n}<\alpha<1$. Then, $\left\{x_{n}\right\}_{n=0}^{\infty}$ converges strongly to $P_{F(T)} x_{0}$.

Motivated by the results of Takahashi et al. [33], Kumam [28] studied the problem of approximating a common element of set of solutions to an equilibrium problem, set of solutions to variational inequality problem and the set of fixed points of a nonexpansive mapping in a real Hilbert space. In particular, he proved the following theorem.

Theorem 1.2 (Kumam, [28]). Let $K$ be a nonempty closed convex subset of a real Hilbert space $H$. Let $F$ be a bifunction from $K \times K$ satisfying (A1)-(A4) and let $B$ be a $\beta$-inverse-strongly monotone mapping of $K$ into $H$. Let $T$ be a nonexpansive mapping of $K$ into $H$ such that $F(T) \cap \operatorname{EP}(F) \cap \operatorname{VI}(K, B) \neq \emptyset$. For $C_{1}=K, x_{1}=P_{C_{1}} x_{0}$, define sequences $\left\{x_{n}\right\}_{n=0}^{\infty}$ and $\left\{z_{n}\right\}_{n=1}^{\infty}$ of $K$ as follows:

$$
\begin{gathered}
F\left(z_{n}, y\right)+\frac{1}{r_{n}}\left\langle y-z_{n}, z_{n}-x_{n}\right\rangle \geq 0, \quad \forall y \in K, \\
y_{n}=\alpha_{n} x_{n}+\left(1-\alpha_{n}\right) T P_{K}\left(z_{n}-\lambda_{n} B z_{n}\right), \quad n \geq 1, \\
C_{n+1}=\left\{z \in C_{n}:\left\|y_{n}-z\right\| \leq\left\|x_{n}-z\right\|\right\}, \quad n \geq 1, \\
x_{n+1}=P_{C_{n+1}} x_{0}, \quad n \geq 1 .
\end{gathered}
$$

Assume that $\left\{\alpha_{n}\right\}_{n=1}^{\infty} \subset[0,1),\left\{r_{n}\right\}_{n=1}^{\infty} \subset(0, \infty)$ and $\left\{\lambda_{n}\right\}_{n=1}^{\infty} \subset[0,2 \beta]$ satisfy

$$
\liminf _{n \rightarrow \infty} r_{n}>0, \quad 0<c \leq \lambda_{n} \leq f<2 \beta, \quad \lim _{n \rightarrow \infty} \alpha_{n}=0 .
$$

Then, $\left\{x_{n}\right\}_{n=0}^{\infty}$ converges strongly to $P_{F(T) \cap \operatorname{EP}(F) \cap \mathrm{VI}(K, B)} x_{0}$.

Motivated by the ongoing research and the above-mentioned results, we introduce $a$ new iterative scheme for finding a common element of the set of fixed points of an infinite family of $k$-strictly pseudocontractive mappings, the set of common solutions to a system of generalized mixed equilibrium problems and the set of solutions to a variational inequality problem in a real Hilbert space. Furthermore, we show that our new iterative scheme converges strongly to a common 
element of the three afore mentioned sets. In our results, we use conditions (B1) and (B2) mentioned above. Our result extends many important recent results. Finally, we give some applications of our results.

\section{Preliminaries}

Let $H$ be a real Hilbert space with inner product $\langle\cdot, \cdot\rangle$ and norm $\|\cdot\|$ and let $K$ be a nonempty closed and convex subset of $H$. The strong convergence of $\left\{x_{n}\right\}_{n=0}^{\infty}$ to $x$ is denoted by $x_{n} \rightarrow x$ as $n \rightarrow \infty$.

For any point $u \in H$, there exists a unique point $P_{K} u \in K$ such that

$$
\left\|u-P_{K} u\right\| \leq\|u-y\|, \quad \forall y \in K
$$

$P_{K}$ is called the metric projection of $H$ onto $K$. We know that $P_{K}$ is a nonexpansive mapping of $H$ onto $K$. It is also known that $P_{K}$ satisfies

$$
\left\langle x-y, P_{K} x-P_{K} y\right\rangle \geq\left\|P_{K} x-P_{K} y\right\|^{2}
$$

for all $x, y \in H$. Furthermore, $P_{K} x$ is characterized by the properties $P_{K} x \in K$ and

$$
\left\langle x-P_{K} x, P_{K} x-y\right\rangle \geq 0,
$$

for all $y \in K$ and

$$
\left\|x-P_{K} x\right\|^{2} \leq\|x-y\|^{2}-\left\|y-P_{K} x\right\|^{2}, \quad \forall x \in H, y \in K .
$$

In the context of the variational inequality problem, (2.3) implies that

$$
x^{*} \in \mathrm{VI}(A, K) \Longleftrightarrow x^{*}=P_{K}\left(x^{*}-\lambda A x^{*}\right), \quad \forall \lambda>0 .
$$

If $A$ is $\alpha$-inverse-strongly monotone mapping of $K$ into $H$, then it is obvious that $A$ is $(1 / \alpha)$ Lipschitz continuous. We also have that for all $x, y \in K$ and $r>0$,

$$
\begin{aligned}
\|(I-r A) x-(I-r A) y\|^{2} & =\|x-y-r(A x-A y)\|^{2} \\
& =\|x-y\|^{2}-2 r\langle A x-A y, x-y\rangle+r^{2}\|A x-A y\|^{2} \\
& \leq\|x-y\|^{2}+r(r-2 \alpha)\|A x-A y\|^{2} .
\end{aligned}
$$

So, if $r \leq 2 \alpha$, then $I-r A$ is a nonexpansive mapping of $K$ into $H$.

For solving the generalized mixed equilibrium problem for a bifunction $F: K \times K \rightarrow$ $\mathbb{R}$, let us assume that $F$ satisfies the following conditions:

(A1) $F(x, x)=0$ for all $x \in K$, 
(A2) $F$ is monotone, that is, $F(x, y)+F(y, x) \leq 0$ for all $x, y \in K$,

(A3) for each $x, y, z \in K, \lim _{t \rightarrow 0} F(t z+(1-t) x, y) \leq F(x, y)$,

(A4) for each $x \in K, y \mapsto F(x, y)$ is convex and lower semicontinuous.

We need the following technical result.

Lemma 2.1 (R. Wangkeeree and R. Wangkeeree [30]). Assume that $F: K \times K \rightarrow \mathbb{R}$ satisfies (A1)-(A4) and let $\varphi: K \rightarrow \mathbb{R}$ be a proper lower semicontinuous and convex function. Assume that either (B1) or (B2) holds. For $r>0$ and $x \in H$, define a mapping $T_{r}^{(F, \varphi)}: H \rightarrow K$ as follows:

$$
T_{r}^{(F, \varphi)}(x)=\left\{z \in K: F(z, y)+\varphi(y)-\varphi(z)+\frac{1}{r}\langle y-z, z-x\rangle \geq 0, \forall y \in K\right\}
$$

for all $z \in H$. Then, the following hold:

(1) for each $x \in H, T_{r}^{(F, \varphi)} \neq \emptyset$,

(2) $T_{r}^{(F, \varphi)}$ is single-valued,

(3) $T_{r}^{(F, \varphi)}$ is firmly nonexpansive, that is, for any $x, y \in H$,

$$
\left\|T_{r}^{(F, \varphi)} x-T_{r}^{(F, \varphi)} y\right\| \leq\left\langle T_{r}^{(F, \varphi)} x-T_{r}^{(F, \varphi)} y, x-y\right\rangle,
$$

(4) $F\left(T_{r}^{(F, \varphi)}\right)=\operatorname{MEP}(F)$,

(5) $\operatorname{MEP}(F)$ is closed and convex.

\section{Main Results}

Theorem 3.1. Let $K$ be a nonempty closed and convex subset of a real Hilbert space $H$. For each $m=1,2$, let $F_{m}$ be a bifunction from $K \times K$ satisfying $(A 1)-(A 4), \varphi_{m}: K \rightarrow \mathbb{R} \cup\{+\infty\}$ a proper lower semicontinuous and convex function with assumption (B1) or (B2), $A$ an $\alpha$-inverse-strongly monotone mapping of $K$ into $H, B$ a $\beta$-inverse-strongly monotone mapping of $K$ into $H$ and for each $i=1,2, \ldots$, let $T_{i}: K \rightarrow K$ be a $k_{i}$-strictly pseudocontractive mapping for some $0 \leq k_{i}<1$ such that $\cap_{i=1}^{\infty} F\left(T_{i}\right) \neq \emptyset$. Let $D$ be a $\mu$-Lipschitzian, relaxed $(\lambda, \gamma)$-cocoercive mapping of $K$ into $H$. Suppose $\Omega:=\cap_{i=1}^{\infty} F\left(T_{i}\right) \cap \operatorname{GMEP}\left(F_{1}, A, \varphi_{1}\right) \cap \operatorname{GMEP}\left(F_{2}, B, \varphi_{2}\right) \cap \operatorname{VI}(K, D) \neq \emptyset$. Let $\left\{z_{n}\right\}_{n=1}^{\infty},\left\{u_{n}\right\}_{n=1}^{\infty}$, 
$\left\{w_{n}\right\}_{n=1}^{\infty},\left\{y_{n, i}\right\}_{n=1}^{\infty}(i=1,2, \ldots)$ and $\left\{x_{n}\right\}_{n=0}^{\infty}$ be generated by $x_{0} \in K, C_{1, i}=K, C_{1}=\cap_{i=1}^{\infty} C_{1, i}$, $x_{1}=P_{C_{1}} x_{0}$

$$
\begin{gathered}
z_{n}=T_{r_{n}}^{\left(F_{1}, \varphi_{1}\right)}\left(x_{n}-r_{n} A x_{n}\right), \\
u_{n}=T_{\lambda_{n}}^{\left(F_{2}, \varphi_{2}\right)}\left(z_{n}-\lambda_{n} B z_{n}\right), \\
w_{n}=P_{K}\left(u_{n}-s_{n} D u_{n}\right), \\
y_{n, i}=\alpha_{n, i} w_{n}+\left(1-\alpha_{n, i}\right) T_{i} w_{n}, \\
C_{n+1, i}=\left\{z \in C_{n, i}:\left\|y_{n, i}-z\right\| \leq\left\|x_{n}-z\right\|\right\}, \\
C_{n+1}=\cap_{i=1}^{\infty} C_{n+1, i} \\
x_{n+1}=P_{C_{n+1}} x_{0}, \quad n \geq 1 .
\end{gathered}
$$

Assume that $\left\{\alpha_{n, i}\right\}_{n=1}^{\infty} \subset[0,1)(i=1,2, \ldots),\left\{r_{n}\right\}_{n=1}^{\infty} \subset[0,2 \alpha]$ and $\left\{\lambda_{n}\right\}_{n=1}^{\infty} \subset[0,2 \beta]$ satisfy

(i) $0<a \leq r_{n} \leq b<2 \alpha$,

(ii) $0<c \leq \lambda_{n} \leq f<2 \beta$,

(iii) $0 \leq k_{i} \leq \alpha_{n, i} \leq d_{i}<1$,

(iv) $0<h \leq s_{n} \leq j<2\left(\gamma-\lambda \mu^{2}\right) / \mu^{2}$.

Then, $\left\{x_{n}\right\}_{n=0}^{\infty}$ converges strongly to $P_{\Omega} x_{0}$.

Proof. For all $x, y \in K$ and $s_{n} \in\left(0,2\left(\gamma-\lambda \mu^{2}\right) / \mu^{2}\right]$, we obtain

$$
\begin{aligned}
\left\|\left(I-s_{n} D\right) x-\left(I-s_{n} D\right) y\right\|^{2} & =\left\|x-y-s_{n}(D x-D y)\right\|^{2} \\
& =\|x-y\|^{2}-2 s_{n}\langle x-y, D x-D y\rangle+s_{n}^{2}\|D x-D y\|^{2} \\
& \leq\|x-y\|^{2}-2 s_{n}\left[-\lambda\|D x-D y\|^{2}+\gamma\|x-y\|^{2}\right]+s_{n}^{2}\|D x-D y\|^{2} \\
& \leq\|x-y\|^{2}+2 s_{n} \mu^{2} \lambda\|x-y\|^{2}-2 s_{n} \gamma\|x-y\|^{2}+\mu^{2} s_{n}^{2}\|x-y\|^{2} \\
& =\left(1+2 s_{n} \mu^{2} \lambda-2 s_{n} \gamma+\mu^{2} s_{n}^{2}\right)\|x-y\|^{2} \\
& \leq\|x-y\|^{2} .
\end{aligned}
$$

This shows that $I-s_{n} D$ is nonexpansive for each $n \geq 1$. Let $x^{*} \in \Omega$. Then

$$
\begin{aligned}
\left\|w_{n}-x^{*}\right\|^{2} & =\left\|P_{K}\left(u_{n}-s_{n} D u_{n}\right)-P_{K}\left(x^{*}-s_{n} D x^{*}\right)\right\|^{2} \\
& \leq\left\|\left(u_{n}-s_{n} D u_{n}\right)-\left(x^{*}-s_{n} D x^{*}\right)\right\|^{2} \\
& \leq\left\|u_{n}-x^{*}\right\|^{2} .
\end{aligned}
$$


Since both $I-r_{n} A$ and $I-\lambda_{n} B$ are nonexpansive for each $n \geq 1$ and $x^{*}=T_{r_{n}}^{\left(F_{1}, \varphi_{1}\right)}\left(x^{*}-r_{n} A x^{*}\right), x^{*}=$ $T_{\lambda_{n}}^{\left(F_{2}, \varphi_{2}\right)}\left(x^{*}-\lambda_{n} B x^{*}\right)$, from (2.6), we have

$$
\begin{aligned}
\left\|u_{n}-x^{*}\right\|^{2} & =\left\|T_{\lambda_{n}}^{\left(F_{2}, \varphi_{2}\right)}\left(z_{n}-\lambda_{n} B z_{n}\right)-x^{*}\right\|^{2} \\
& =\left\|T_{\lambda_{n}}^{\left(F_{2}, \varphi_{2}\right)}\left(z_{n}-\lambda_{n} B z_{n}\right)-T_{\lambda_{n}}^{\left(F_{2}, \varphi_{2}\right)}\left(x^{*}-\lambda_{n} B x^{*}\right)\right\|^{2} \\
& \leq\left\|\left(I-\lambda_{n} B\right) z_{n}-\left(I-\lambda_{n} B\right) x^{*}\right\|^{2} \\
& \leq\left\|z_{n}-x^{*}\right\|^{2}+\lambda_{n}\left(\lambda_{n}-2 \beta\right)\left\|B z_{n}-B x^{*}\right\|^{2} \\
& \leq\left\|z_{n}-x^{*}\right\|^{2} \quad\left(\text { since } \lambda_{n}<2 \beta, \forall n \geq 1\right), \\
\left\|z_{n}-x^{*}\right\|^{2} & =\left\|T_{r_{n}}^{\left(F_{1}, \varphi_{1}\right)}\left(x_{n}-r_{n} A x_{n}\right)-x^{*}\right\|^{2} \\
& =\left\|T_{r_{n}}^{\left(F_{1}, \varphi_{1}\right)}\left(x_{n}-r_{n} A x_{n}\right)-T_{r_{n}}^{\left(F_{1}, \varphi_{1}\right)}\left(x^{*}-r_{n} A x^{*}\right)\right\|^{2} \\
& \leq\left\|\left(I-r_{n} A\right) x_{n}-\left(I-r_{n} A\right) x^{*}\right\|^{2} \\
& \leq\left\|x_{n}-x^{*}\right\|^{2}+r_{n}\left(r_{n}-2 \alpha\right)\left\|A x_{n}-A x^{*}\right\|^{2} \\
& \leq\left\|x_{n}-x^{*}\right\|^{2} .
\end{aligned}
$$

Therefore,

$$
\left\|u_{n}-x^{*}\right\| \leq\left\|x_{n}-x^{*}\right\|
$$

Let $n=1$, then $C_{1, i}=K$ is closed convex for each $i=1,2, \ldots$. Now assume that $C_{n, i}$ is closed convex for some $n>1$. Then, from definition of $C_{n+1, i}$, we know that $C_{n+1, i}$ is closed convex for the same $n>1$. Hence, $C_{n, i}$ is closed convex for $n \geq 1$ and for each $i=1,2, \ldots$. This implies that $C_{n}$ is closed convex for $n \geq 1$. Furthermore, we show that $\Omega \subset C_{n}$. For $n=1, \Omega \subset K=C_{1, i}$. For $n \geq 2$, let $x^{*} \in \Omega$. Then,

$$
\begin{aligned}
\left\|y_{n, i}-x^{*}\right\|^{2}= & \alpha_{n, i}\left\|w_{n}-x^{*}\right\|^{2}+\left(1-\alpha_{n, i}\right)\left\|T_{i} w_{n}-x^{*}\right\|^{2}-\alpha_{n, i}\left(1-\alpha_{n, i}\right)\left\|T_{i} w_{n}-w_{n}\right\|^{2} \\
\leq & \alpha_{n, i}\left\|w_{n}-x^{*}\right\|^{2}+\left(1-\alpha_{n, i}\right)\left[\left\|w_{n}-x^{*}\right\|^{2}+k_{i}\left\|T_{i} w_{n}-w_{n}\right\|^{2}\right] \\
& -\alpha_{n, i}\left(1-\alpha_{n, i}\right)\left\|T_{i} w_{n}-w_{n}\right\|^{2} \\
= & \left\|w_{n}-x^{*}\right\|^{2}+\left(1-\alpha_{n, i}\right)\left(k_{i}-\alpha_{n, i}\right)\left\|T_{i} w_{n}-w_{n}\right\|^{2} \\
\leq & \left\|u_{n}-x^{*}\right\|^{2} \leq\left\|x_{n}-x^{*}\right\|^{2}
\end{aligned}
$$


which shows that $x^{*} \in C_{n, i}$, for all $n \geq 2$, for all $i=1,2, \ldots$. Thus, $\Omega \subset C_{n, i}$, for all $n \geq 1$, for all $i=1,2, \ldots$. Hence, it follows that $\emptyset \neq \Omega \subset C_{n}$, for all $n \geq 1$. Therefore, $\left\{x_{n}\right\}_{n=0}^{\infty}$ is well defined. Since $x_{n}=P_{C_{n}} x_{0}$, for all $n \geq 1$ and $x_{n+1} \in C_{n+1} \subset C_{n}$, for all $n \geq 1$, we have

$$
\left\|x_{n}-x_{0}\right\| \leq\left\|x_{n+1}-x_{0}\right\|, \quad \forall n \geq 1
$$

Also, as $\Omega \subset C_{n}$, by (2.1) it follows that

$$
\left\|x_{n}-x_{0}\right\| \leq\left\|v-x_{0}\right\|, \quad v \in \Omega, \forall n \geq 1
$$

From (3.7) and (3.8), we have that $\lim _{n \rightarrow \infty}\left\|x_{n}-x_{0}\right\|$ exists. Hence, $\left\{x_{n}\right\}_{n=0}^{\infty}$ is bounded and so are $\left\{z_{n}\right\}_{n=1}^{\infty},\left\{A x_{n}\right\}_{n=1}^{\infty},\left\{u_{n}\right\}_{n=1}^{\infty},\left\{D u_{n}\right\}_{n=1}^{\infty},\left\{B z_{n}\right\}_{n=1}^{\infty},\left\{w_{n}\right\}_{n=1}^{\infty},\left\{T_{i} w_{n}\right\}_{n=1}^{\infty}$ and $\left\{y_{n, i}\right\}_{n=1}^{\infty}, i=$ $1,2, \ldots$. For $m>n \geq 1$, we have that $x_{m}=P_{C_{m}} x_{0} \in C_{m} \subset C_{n}$. By (2.4), we obtain

$$
\left\|x_{m}-x_{n}\right\|^{2} \leq\left\|x_{m}-x_{0}\right\|^{2}-\left\|x_{n}-x_{0}\right\|^{2}
$$

Letting $m, n \rightarrow \infty$ and taking the limit in (3.9), we have $x_{m}-x_{n} \rightarrow 0, m, n \rightarrow \infty$, which shows that $\left\{x_{n}\right\}_{n=0}^{\infty}$ is Cauchy. In particular, $\lim _{n \rightarrow \infty}\left\|x_{n+1}-x_{n}\right\|=0$. Since, $\left\{x_{n}\right\}_{n=0}^{\infty}$ is Cauchy and $K$ is closed, there exists $z \in K$ such that $x_{n} \rightarrow z, n \rightarrow \infty$. Since $x_{n+1}=P_{C_{n+1}} x_{0} \in C_{n+1}$, therefore

$$
\left\|y_{n, i}-x_{n+1}\right\| \leq\left\|x_{n}-x_{n+1}\right\|,
$$

and it follows that

$$
\begin{aligned}
\left\|y_{n, i}-x_{n}\right\| & \leq\left\|y_{n, i}-x_{n+1}\right\|+\left\|x_{n}-x_{n+1}\right\| \\
& \leq 2\left\|x_{n}-x_{n+1}\right\| .
\end{aligned}
$$

Thus,

$$
\lim _{n \rightarrow \infty}\left\|y_{n, i}-x_{n}\right\|=0, \quad i=1,2, \ldots
$$


Furthermore,

$$
\begin{aligned}
\left\|y_{n, i}-x^{*}\right\|^{2}= & \alpha_{n, i}\left\|w_{n}-x^{*}\right\|^{2}+\left(1-\alpha_{n, i}\right)\left\|T_{i} w_{n}-x^{*}\right\|^{2} \\
& -\alpha_{n, i}\left(1-\alpha_{n, i}\right)\left\|T_{i} w_{n}-w_{n}\right\|^{2} \\
\leq & \alpha_{n, i}\left\|w_{n}-x^{*}\right\|^{2}+\left(1-\alpha_{n, i}\right)\left[\left\|w_{n}-x^{*}\right\|^{2}+k_{i}\left\|T_{i} w_{n}-w_{n}\right\|^{2}\right] \\
& -\alpha_{n, i}\left(1-\alpha_{n, i}\right)\left\|T_{i} w_{n}-w_{n}\right\|^{2} \\
= & \alpha_{n, i}\left\|w_{n}-x^{*}\right\|^{2}+\left(1-\alpha_{n, i}\right)\left\|w_{n}-x^{*}\right\|^{2} \\
& -\left(1-\alpha_{n, i}\right)\left(\alpha_{n, i}-k_{i}\right)\left\|T_{i} w_{n}-w_{n}\right\|^{2} \\
\leq & \alpha_{n, i}\left\|u_{n}-x^{*}\right\|^{2}+\left(1-\alpha_{n, i}\right)\left\|u_{n}-x^{*}\right\|^{2} \\
\leq & \alpha_{n, i}\left\|u_{n}-x^{*}\right\|^{2}+\left(1-\alpha_{n, i}\right)\left\|T_{\lambda_{n}}^{\left(F_{2}, \varphi_{2}\right)}\left(z_{n}-\lambda_{n} B z_{n}\right)-T_{\lambda_{n}}^{\left(F_{2}, \varphi_{2}\right)}\left(x^{*}-\lambda_{n} B x^{*}\right)\right\|^{2} \\
\leq & \alpha_{n, i}\left\|u_{n}-x^{*}\right\|^{2}+\left(1-\alpha_{n, i}\right)\left\|\left(z_{n}-\lambda_{n} B z_{n}\right)-\left(x^{*}-\lambda_{n} B x^{*}\right)\right\|^{2} \\
\leq & \alpha_{n, i}\left\|u_{n}-x^{*}\right\|^{2}+\left(1-\alpha_{n, i}\right)\left[\left\|z_{n}-x^{*}\right\|^{2}+\lambda_{n}\left(\lambda_{n}-2 \beta\right)\left\|B z_{n}-B x^{*}\right\|^{2}\right] \\
\leq & \left\|u_{n}-x^{*}\right\|^{2}+\left(1-\alpha_{n, i}\right) \lambda_{n}\left(\lambda_{n}-2 \beta\right)\left\|B z_{n}-B x^{*}\right\|^{2} \\
\leq & \left\|x_{n}-x^{*}\right\|^{2}+\left(1-\alpha_{n, i}\right) \lambda_{n}\left(\lambda_{n}-2 \beta\right)\left\|B z_{n}-B x^{*}\right\|^{2} .
\end{aligned}
$$

Since $0<c \leq \lambda_{n} \leq f<2 \beta, 0 \leq k_{i} \leq \alpha_{n, i} \leq d_{i}<1$, we have

$$
\begin{aligned}
\left(1-d_{i}\right) c(2 \beta-f)\left\|B z_{n}-B x^{*}\right\|^{2} & \leq\left\|x_{n}-x^{*}\right\|^{2}-\left\|y_{n, i}-x^{*}\right\|^{2} \\
& \leq\left\|y_{n, i}-x_{n}\right\|\left(\left\|x_{n}-x^{*}\right\|+\left\|y_{n, i}-x^{*}\right\|\right)
\end{aligned}
$$

Hence, $\lim _{n \rightarrow \infty}\left\|B z_{n}-B x^{*}\right\|=0$. From (3.1), we have

$$
\begin{aligned}
\left\|y_{n, i}-x^{*}\right\|^{2} \leq & \alpha_{n, i}\left\|u_{n}-x^{*}\right\|^{2}+\left(1-\alpha_{n, i}\right)\left[\left\|u_{n}-x^{*}\right\|^{2}+k_{i}\left\|T_{i} w_{n}-w_{n}\right\|^{2}\right] \\
& -\alpha_{n, i}\left(1-\alpha_{n, i}\right)\left\|T_{i} w_{n}-w_{n}\right\|^{2} \\
= & \alpha_{n, i}\left\|u_{n}-x^{*}\right\|^{2}+\left(1-\alpha_{n, i}\right)\left\|u_{n}-x^{*}\right\|^{2}-\left(1-\alpha_{n, i}\right)\left(\alpha_{n, i}-k_{i}\right)\left\|T_{i} w_{n}-w_{n}\right\|^{2} \\
\leq & \left\|u_{n}-x^{*}\right\|^{2} .
\end{aligned}
$$


On the other hand,

$$
\begin{aligned}
\left\|u_{n}-x^{*}\right\|^{2} \leq & \left\|T_{\lambda_{n}}^{\left(F_{2}, \varphi_{2}\right)}\left(z_{n}-\lambda_{n} B z_{n}\right)-T_{\lambda_{n}}^{\left(F_{2}, \varphi_{2}\right)}\left(x^{*}-\lambda_{n} B x^{*}\right)\right\|^{2} \\
\leq & \left\langle\left(z_{n}-\lambda_{n} B z_{n}\right)-\left(x^{*}-\lambda_{n} B x^{*}\right), u_{n}-x^{*}\right\rangle \\
= & \frac{1}{2}\left[\left\|\left(z_{n}-\lambda_{n} B z_{n}\right)-\left(x^{*}-\lambda_{n} B x^{*}\right)\right\|^{2}+\left\|u_{n}-x^{*}\right\|^{2}\right. \\
& \left.\quad-\left\|\left(z_{n}-\lambda_{n} B z_{n}\right)-\left(x^{*}-\lambda_{n} B x^{*}\right)-\left(u_{n}-x^{*}\right)\right\|^{2}\right] \\
\leq & \frac{1}{2}\left[\left\|z_{n}-x^{*}\right\|^{2}+\left\|u_{n}-x^{*}\right\|^{2}-\left\|\left(z_{n}-\lambda_{n} B z_{n}\right)-\left(x^{*}-\lambda_{n} B x^{*}\right)-\left(u_{n}-x^{*}\right)\right\|^{2}\right] \\
= & \frac{1}{2}\left[\left\|z_{n}-x^{*}\right\|^{2}+\left\|u_{n}-x^{*}\right\|^{2}-\left\|u_{n}-z_{n}\right\|^{2}+2 \lambda_{n}\left\langle z_{n}-u_{n}, B z_{n}-B x^{*}\right\rangle\right. \\
& \left.\quad-\lambda_{n}^{2}\left\|B z_{n}-B x^{*}\right\|^{2}\right],
\end{aligned}
$$

and hence

$$
\begin{aligned}
\left\|u_{n}-x^{*}\right\|^{2} \leq & \left\|z_{n}-x^{*}\right\|^{2}-\left\|u_{n}-z_{n}\right\|^{2}+2 \lambda_{n}\left\langle z_{n}-u_{n}, B z_{n}-B x^{*}\right\rangle \\
& -\lambda_{n}^{2}\left\|B z_{n}-B x^{*}\right\|^{2} \\
\leq & \left\|z_{n}-x^{*}\right\|^{2}-\left\|u_{n}-z_{n}\right\|^{2}+2 \lambda_{n}\left\|z_{n}-u_{n}\right\|\left\|B z_{n}-B x^{*}\right\| .
\end{aligned}
$$

Putting (3.17) into (3.15), we have

$$
\left\|y_{n, i}-x^{*}\right\|^{2} \leq\left\|z_{n}-x^{*}\right\|^{2}-\left\|u_{n}-z_{n}\right\|^{2}+2 \lambda_{n}\left\|z_{n}-u_{n}\right\|\left\|B z_{n}-B x^{*}\right\| .
$$

It follows that

$$
\begin{aligned}
\left\|z_{n}-u_{n}\right\|^{2} \leq & \left\|z_{n}-x^{*}\right\|^{2}-\left\|y_{n, i}-x^{*}\right\|^{2}+2 \lambda_{n}\left\|z_{n}-u_{n}\right\|\left\|B z_{n}-B x^{*}\right\| \\
\leq & \left\|x_{n}-x^{*}\right\|^{2}-\left\|y_{n, i}-x^{*}\right\|^{2}+2 \lambda_{n}\left\|z_{n}-u_{n}\right\|\left\|B z_{n}-B x^{*}\right\| \\
\leq & \left\|y_{n, i}-x_{n}\right\|\left(\left\|x_{n}-x^{*}\right\|+\left\|y_{n, i}-x^{*}\right\|\right) \\
& +2 \lambda_{n}\left\|z_{n}-u_{n}\right\|\left\|B z_{n}-B x^{*}\right\| .
\end{aligned}
$$


Therefore, $\lim _{n \rightarrow \infty}\left\|z_{n}-u_{n}\right\|=0$. Furthermore,

$$
\begin{aligned}
\left\|y_{n, i}-x^{*}\right\|^{2} \leq & \alpha_{n, i}\left\|w_{n}-x^{*}\right\|^{2}+\left(1-\alpha_{n, i}\right)\left\|T_{i} w_{n}-x^{*}\right\|^{2} \\
& -\alpha_{n, i}\left(1-\alpha_{n, i}\right)\left\|T_{i} w_{n}-w_{n}\right\|^{2} \\
\leq & \alpha_{n, i}\left\|x_{n}-x^{*}\right\|^{2}+\left(1-\alpha_{n, i}\right)\left\|u_{n}-x^{*}\right\|^{2} \\
& -\left(1-\alpha_{n, i}\right)\left(\alpha_{n, i}-k_{i}\right)\left\|T_{i} w_{n}-w_{n}\right\|^{2} \\
\leq & \alpha_{n, i}\left\|x_{n}-x^{*}\right\|^{2}+\left(1-\alpha_{n, i}\right)\left\|u_{n}-x^{*}\right\|^{2} \\
\leq & \alpha_{n, i}\left\|x_{n}-x^{*}\right\|^{2}+\left(1-\alpha_{n, i}\right)\left\|z_{n}-x^{*}\right\|^{2} \\
\leq & \alpha_{n, i}\left\|x_{n}-x^{*}\right\|^{2}+\left(1-\alpha_{n, i}\right)\left\|T_{r_{n}}^{\left(F_{1}, \varphi_{1}\right)}\left(x_{n}-r_{n} A x_{n}\right)-T_{r_{n}}^{\left(F_{1}, \varphi_{1}\right)}\left(x^{*}-r_{n} A x^{*}\right)\right\|^{2} \\
\leq & \alpha_{n, i}\left\|x_{n}-x^{*}\right\|^{2}+\left(1-\alpha_{n, i}\right)\left\|\left(x_{n}-r_{n} A x_{n}\right)-\left(x^{*}-r_{n} A x^{*}\right)\right\|^{2} \\
\leq & \alpha_{n, i}\left\|x_{n}-x^{*}\right\|^{2}+\left(1-\alpha_{n, i}\right)\left[\left\|x_{n}-x^{*}\right\|^{2}+r_{n}\left(r_{n}-2 \alpha\right)\left\|A x_{n}-A x^{*}\right\|^{2}\right] \\
= & \left\|x_{n}-x^{*}\right\|^{2}+\left(1-\alpha_{n, i}\right) r_{n}\left(r_{n}-2 \alpha\right)\left\|A x_{n}-A x^{*}\right\|^{2} .
\end{aligned}
$$

Since $0<a \leq r_{n} \leq b<2 \alpha$ and $0 \leq k_{i} \leq \alpha_{n, i} \leq d_{i}<1$, we have

$$
\begin{aligned}
\left(1-d_{i}\right) a(2 \alpha-b)\left\|A x_{n}-A x^{*}\right\|^{2} & \leq\left\|x_{n}-x^{*}\right\|^{2}-\left\|y_{n, i}-x^{*}\right\|^{2} \\
& \leq\left\|y_{n, i}-x_{n}\right\|\left(\left\|x_{n}-x^{*}\right\|+\left\|y_{n, i}-x^{*}\right\|\right) .
\end{aligned}
$$

Hence, $\lim _{n \rightarrow \infty}\left\|A x_{n}-A x^{*}\right\|=0$. From (3.1), we have

$$
\begin{aligned}
\left\|y_{n, i}-x^{*}\right\|^{2} \leq & \alpha_{n, i}\left\|w_{n}-x^{*}\right\|^{2}+\left(1-\alpha_{n, i}\right)\left\|T_{i} w_{n}-x^{*}\right\|^{2} \\
& -\alpha_{n, i}\left(1-\alpha_{n, i}\right)\left\|T_{i} w_{n}-w_{n}\right\|^{2} \\
\leq & \alpha_{n, i}\left\|x_{n}-x^{*}\right\|^{2}+\left(1-\alpha_{n, i}\right)\left\|u_{n}-x^{*}\right\|^{2} \\
& -\left(1-\alpha_{n, i}\right)\left(\alpha_{n, i}-k_{i}\right)\left\|T_{i} w_{n}-w_{n}\right\|^{2} \\
\leq & \alpha_{n, i}\left\|x_{n}-x^{*}\right\|^{2}+\left(1-\alpha_{n, i}\right)\left\|u_{n}-x^{*}\right\|^{2} \\
\leq & \alpha_{n, i}\left\|x_{n}-x^{*}\right\|^{2}+\left(1-\alpha_{n, i}\right)\left\|z_{n}-x^{*}\right\|^{2} .
\end{aligned}
$$


On the other hand,

$$
\begin{aligned}
&\left\|z_{n}-x^{*}\right\|^{2} \leq\left\|T_{r_{n}}^{\left(F_{1}, \varphi_{1}\right)}\left(x_{n}-r_{n} A x_{n}\right)-T_{r_{n}}^{\left(F_{1}, \varphi_{1}\right)}\left(x^{*}-r_{n} A x^{*}\right)\right\|^{2} \\
& \leq\left\langle\left(x_{n}-r_{n} A x_{n}\right)-\left(x^{*}-r_{n} A x^{*}\right), z_{n}-x^{*}\right\rangle \\
&= \frac{1}{2}\left[\left\|\left(x_{n}-r_{n} A x_{n}\right)-\left(x^{*}-r_{n} A x^{*}\right)\right\|^{2}+\left\|z_{n}-x^{*}\right\|^{2}\right. \\
&\left.\quad-\left\|\left(x_{n}-r_{n} A x_{n}\right)-\left(x^{*}-r_{n} A x^{*}\right)-\left(z_{n}-x^{*}\right)\right\|^{2}\right] \\
& \leq \frac{1}{2}\left[\left\|x_{n}-x^{*}\right\|^{2}+\left\|z_{n}-x^{*}\right\|^{2}-\left\|\left(x_{n}-r_{n} A x_{n}\right)-\left(x^{*}-r_{n} A x^{*}\right)-\left(z_{n}-x^{*}\right)\right\|^{2}\right] \\
&= \frac{1}{2}\left[\left\|x_{n}-x^{*}\right\|^{2}+\left\|z_{n}-x^{*}\right\|^{2}-\left\|z_{n}-x_{n}\right\|^{2}+2 r_{n}\left\langle x_{n}-z_{n}, A x_{n}-A x^{*}\right\rangle\right. \\
&\left.\quad-r_{n}^{2}\left\|A x_{n}-A x^{*}\right\|^{2}\right],
\end{aligned}
$$

and hence

$$
\begin{aligned}
\left\|z_{n}-x^{*}\right\|^{2} & \leq\left\|x_{n}-x^{*}\right\|^{2}-\left\|z_{n}-x_{n}\right\|^{2}+2 r_{n}\left\langle x_{n}-z_{n}, A x_{n}-A x^{*}\right\rangle-r_{n}^{2}\left\|A x_{n}-A x^{*}\right\|^{2} \\
& \leq\left\|x_{n}-x^{*}\right\|^{2}-\left\|z_{n}-x_{n}\right\|^{2}+2 r_{n}\left\|x_{n}-z_{n}\right\|\left\|A x_{n}-A x^{*}\right\| .
\end{aligned}
$$

Putting (3.24) into (3.22), we have

$$
\left\|y_{n, i}-x^{*}\right\|^{2} \leq\left\|x_{n}-x^{*}\right\|^{2}-\left(1-\alpha_{n, i}\right)\left\|z_{n}-x_{n}\right\|^{2}+2 r_{n}\left\|x_{n}-z_{n}\right\|\left\|A x_{n}-A x^{*}\right\| .
$$

It follows that

$$
\begin{aligned}
\left(1-d_{i}\right)\left\|x_{n}-z_{n}\right\|^{2} & \leq\left\|x_{n}-x^{*}\right\|^{2}-\left\|y_{n, i}-x^{*}\right\|^{2}+2 r_{n}\left\|x_{n}-z_{n}\right\|\left\|A x_{n}-A x^{*}\right\| \\
& \leq\left\|y_{n, i}-x_{n}\right\|\left(\left\|x_{n}-x^{*}\right\|+\left\|y_{n, i}-x^{*}\right\|\right)+2 r_{n}\left\|x_{n}-z_{n}\right\|\left\|A x_{n}-A x^{*}\right\| .
\end{aligned}
$$

Therefore, $\lim _{n \rightarrow \infty}\left\|x_{n}-z_{n}\right\|=0$. Then, we obtain that

$$
\left\|x_{n+1}-u_{n}\right\| \leq\left\|x_{n+1}-x_{n}\right\|+\left\|x_{n}-z_{n}\right\|+\left\|z_{n}-u_{n}\right\| \longrightarrow 0, \quad n \longrightarrow \infty
$$

Since $x_{n+1} \in C_{n+1}$, then

$$
\left\|y_{n, i}-x_{n+1}\right\| \leq\left\|x_{n}-x_{n+1}\right\|
$$

But $y_{n, i}=\alpha_{n, i} w_{n}+\left(1-\alpha_{n, i}\right) T_{i} w_{n}$ implies that

$$
\left\|y_{n, i}-x_{n+1}\right\|^{2}=\alpha_{n, i}\left\|w_{n}-x_{n+1}\right\|^{2}+\left(1-\alpha_{n, i}\right)\left\|T_{i} w_{n}-x_{n+1}\right\|^{2}-\alpha_{n, i}\left(1-\alpha_{n, i}\right)\left\|w_{n}-T_{i} w_{n}\right\|^{2} .
$$


Putting (3.29) into (3.28), we have

$$
\left(1-\alpha_{n, i}\right)\left\|T_{i} w_{n}-x_{n+1}\right\|^{2} \leq \alpha_{n, i}\left(1-\alpha_{n, i}\right)\left\|w_{n}-T_{i} w_{n}\right\|^{2}+\left\|x_{n}-x_{n+1}\right\|^{2}-\alpha_{n, i}\left\|w_{n}-x_{n+1}\right\|^{2} .
$$

Thus, we get

$$
\begin{aligned}
\left\|T_{i} w_{n}-x_{n+1}\right\|^{2} & \leq \alpha_{n, i}\left\|w_{n}-T_{i} w_{n}\right\|^{2}+\frac{1}{\left(1-\alpha_{n, i}\right)}\left\|x_{n}-x_{n+1}\right\|^{2} \\
& \leq \alpha_{n, i}\left\|w_{n}-T_{i} w_{n}\right\|^{2}+\frac{1}{1-d_{i}}\left\|x_{n}-x_{n+1}\right\|^{2} .
\end{aligned}
$$

But

$$
\left\|T_{i} w_{n}-x_{n+1}\right\|^{2}=\left\|x_{n+1}-w_{n}\right\|^{2}+2\left\langle x_{n+1}-w_{n}, w_{n}-T_{i} w_{n}\right\rangle+\left\|w_{n}-T_{i} w_{n}\right\|^{2} .
$$

Putting (3.32) into (3.31) and rearranging, we have

$$
\begin{aligned}
\left(1-d_{i}\right)\left\|w_{n}-T_{i} w_{n}\right\|^{2} & \leq \frac{1}{1-d_{i}}\left\|x_{n}-x_{n+1}\right\|^{2}-2\left\langle x_{n+1}-w_{n}, w_{n}-T_{i} w_{n}\right\rangle \\
& \leq \frac{1}{1-d_{i}}\left\|x_{n}-x_{n+1}\right\|^{2}+2\left\|x_{n+1}-w_{n}\right\|\left\|w_{n}-T_{i} w_{n}\right\| .
\end{aligned}
$$

Hence, $\lim _{n \rightarrow \infty}\left\|w_{n}-T_{i} w_{n}\right\|=0, i=1,2, \ldots$. Now,

$$
\begin{aligned}
\left\|w_{n}-x^{*}\right\|^{2} & \leq\left\|\left(I-s_{n} D\right) u_{n}-\left(I-s_{n} D\right) x^{*}\right\|^{2} \\
& =\left\|u_{n}-x^{*}\right\|^{2}-2 s_{n}\left\langle u_{n}-x^{*}, D u_{n}-D x^{*}\right\rangle+s_{n}^{2}\left\|D u_{n}-D x^{*}\right\|^{2} \\
& \leq\left\|x_{n}-x^{*}\right\|^{2}-2 s_{n}\left[-\lambda\left\|D u_{n}-D x^{*}\right\|^{2}+\gamma\left\|u_{n}-x^{*}\right\|^{2}\right]+s_{n}^{2}\left\|D u_{n}-D x^{*}\right\|^{2} \\
& \leq\left\|x_{n}-x^{*}\right\|^{2}+\left(2 s_{n} \lambda+s_{n}^{2}-\frac{2 s_{n} \gamma}{\mu^{2}}\right)\left\|D u_{n}-D x^{*}\right\|^{2} .
\end{aligned}
$$

Furthermore,

$$
\begin{aligned}
\left\|y_{n, i}-x^{*}\right\|^{2} & \leq \alpha_{n, i}\left\|w_{n}-x^{*}\right\|^{2}+\left(1-\alpha_{n, i}\right)\left\|T_{i} w_{n}-x^{*}\right\|^{2} \\
& \leq \alpha_{n, i}\left\|x_{n}-x^{*}\right\|^{2}+\left(1-\alpha_{n, i}\right)\left\|T_{i} w_{n}-x^{*}\right\|^{2} \\
& \leq \alpha_{n, i}\left\|x_{n}-x^{*}\right\|^{2}+\left(1-\alpha_{n, i}\right)\left\|w_{n}-x^{*}\right\|^{2} \\
& \leq \alpha_{n, i}\left\|x_{n}-x^{*}\right\|^{2}+\left(1-\alpha_{n, i}\right)\left[\left\|x_{n}-x^{*}\right\|^{2}+\left(2 s_{n} \lambda+s_{n}^{2}-\frac{2 s_{n} \gamma}{\mu^{2}}\right)\left\|D u_{n}-D x^{*}\right\|^{2}\right] .
\end{aligned}
$$


Thus,

$$
\begin{aligned}
-\left(2 s_{n} \lambda+s_{n}^{2}-\frac{2 s_{n} \gamma}{\mu^{2}}\right)\left\|D u_{n}-D x^{*}\right\|^{2} & \leq\left\|x_{n}-x^{*}\right\|^{2}-\left\|y_{n, i}-x^{*}\right\|^{2} \\
& \leq\left\|y_{n, i}-x_{n}\right\|\left(\left\|x_{n}-x^{*}\right\|+\left\|y_{n, i}-x^{*}\right\|\right) .
\end{aligned}
$$

By conditions (iii) and (iv), we have that $\lim _{n \rightarrow \infty}\left\|D u_{n}-D x^{*}\right\|=0$. Now, (2.2), we obtain

$$
\begin{aligned}
\left\|w_{n}-x^{*}\right\|^{2} \leq & \left\|P_{K}\left(u_{n}-s_{n} D u_{n}\right)-P_{K}\left(x^{*}-s_{n} D x^{*}\right)\right\|^{2} \\
\leq & \left\langle\left(u_{n}-s_{n} D u_{n}\right)-\left(x^{*}-s_{n} D x^{*}\right), w_{n}-x^{*}\right\rangle \\
= & \frac{1}{2}\left[\left\|\left(u_{n}-s_{n} D u_{n}\right)-\left(x^{*}-s_{n} D x^{*}\right)\right\|^{2}+\left\|w_{n}-x^{*}\right\|^{2}\right. \\
& \left.\quad-\left\|\left(u_{n}-s_{n} D u_{n}\right)-\left(x^{*}-s_{n} D x^{*}\right)-\left(w_{n}-x^{*}\right)\right\|^{2}\right] \\
\leq & \frac{1}{2}\left[\left\|w_{n}-x^{*}\right\|^{2}+\left\|u_{n}-x^{*}\right\|^{2}-\left\|\left(u_{n}-s_{n} D u_{n}\right)-\left(x^{*}-s_{n} D x^{*}\right)-\left(w_{n}-x^{*}\right)\right\|^{2}\right] \\
= & \frac{1}{2}\left[\left\|x_{n}-x^{*}\right\|^{2}+\left\|w_{n}-x^{*}\right\|^{2}-\left\|w_{n}-u_{n}\right\|^{2}+2 s_{n}\left\langle u_{n}-w_{n}, D u_{n}-D x^{*}\right\rangle\right.
\end{aligned}
$$

Thus,

$$
\left\|w_{n}-x^{*}\right\|^{2} \leq\left\|x_{n}-x^{*}\right\|^{2}-\left\|w_{n}-u_{n}\right\|^{2}+2 s_{n}\left\|w_{n}-u_{n}\right\|\left\|D u_{n}-D x^{*}\right\| .
$$

Using this last inequality, we obtain from (3.1) that

$$
\begin{aligned}
\left\|y_{n, i}-x^{*}\right\|^{2} \leq & \alpha_{n, i}\left\|x_{n}-x^{*}\right\|^{2}+\left(1-\alpha_{n, i}\right)\left\|T_{i} w_{n}-x^{*}\right\|^{2} \\
\leq & \alpha_{n, i}\left\|x_{n}-x^{*}\right\|^{2}+\left(1-\alpha_{n, i}\right)\left\|w_{n}-x^{*}\right\|^{2} \\
\leq & \left\|x_{n}-x^{*}\right\|^{2}-\left(1-\alpha_{n, i}\right)\left\|w_{n}-u_{n}\right\|^{2} \\
& +2 s_{n}\left(1-\alpha_{n, i}\right)\left\|w_{n}-u_{n}\right\|\left\|D u_{n}-D x^{*}\right\| .
\end{aligned}
$$

This implies that

$$
\begin{aligned}
\left(1-\alpha_{n, i}\right)\left\|w_{n}-u_{n}\right\|^{2} \leq & \left\|x_{n}-x^{*}\right\|^{2}-\left\|y_{n, i}-x^{*}\right\|^{2} \\
& +2 s_{n}\left(1-\alpha_{n, i}\right)\left\|w_{n}-u_{n}\right\|\left\|D u_{n}-D x^{*}\right\| \\
\leq & \left\|y_{n, i}-x_{n}\right\|\left(\left\|x_{n}-x^{*}\right\|+\left\|y_{n, i}-x^{*}\right\|\right) \\
& +2 s_{n}\left(1-\alpha_{n, i}\right)\left\|w_{n}-u_{n}\right\|\left\|D u_{n}-D x^{*}\right\| .
\end{aligned}
$$


Since $0 \leq k_{i} \leq \alpha_{n, i} \leq d_{i}<1$, we have $\lim _{n \rightarrow \infty}\left\|w_{n}-u_{n}\right\|=0$. Also since $\lim _{n \rightarrow \infty}\left\|w_{n}-x_{n}\right\|=0$ and $\lim _{n \rightarrow \infty}\left\|w_{n}-z\right\|=0$, we have that $\lim _{n \rightarrow \infty}\left\|w_{n}-z\right\|=0$. By $\lim _{n \rightarrow \infty}\left\|w_{n}-z\right\|=0$ and $\lim _{n \rightarrow \infty}\left\|w_{n}-T_{i} w_{n}\right\|=0, i=1,2, \ldots$, we have that $z \in \cap_{i=1}^{\infty} F\left(T_{i}\right)$.Since $z_{n}:=T_{r_{n}}^{\left(F_{1}, \varphi_{1}\right)}\left(x_{n}-r_{n} A x_{n}\right)$, $n \geq 1$, we have for any $y \in K$ that

$$
F_{1}\left(z_{n}, y\right)+\varphi_{1}(y)-\varphi_{1}\left(z_{n}\right)+\left\langle A x_{n}, y-z_{n}\right\rangle+\frac{1}{r_{n}}\left\langle y-z_{n}, z_{n}-x_{n}\right\rangle \geq 0
$$

Furthermore, from the last inequality and using (A2), we obtain

$$
\varphi_{1}(y)-\varphi_{1}\left(z_{n}\right)+\left\langle A x_{n}, y-z_{n}\right\rangle+\frac{1}{r_{n}}\left\langle y-z_{n}, z_{n}-x_{n}\right\rangle \geq F_{1}\left(y, z_{n}\right) .
$$

Let $z_{t}:=t y+(1-t) z$ for all $t \in(0,1]$ and $y \in K$. This implies that $z_{t} \in K$. Then, we have

$$
\begin{aligned}
\left\langle z_{t}-z_{n}, A z_{t}\right\rangle \geq & \varphi_{1}\left(z_{n}\right)-\varphi_{1}\left(z_{t}\right)+\left\langle z_{t}-z_{n}, A z_{t}\right\rangle-\left\langle z_{t}-z_{n}, A x_{n}\right\rangle \\
& -\left\langle z_{t}-z_{n}, \frac{z_{n}-x_{n}}{r_{n}}\right\rangle F_{1}\left(z_{t}, z_{n}\right) \\
= & \varphi_{1}\left(z_{n}\right)-\varphi_{1}\left(z_{t}\right)+\left\langle z_{t}-z_{n}, A z_{t}-A z_{n}\right\rangle \\
& +\left\langle z_{t}-z_{n}, A z_{n}-A x_{n}\right\rangle-\left\langle z_{t}-z_{n}, \frac{z_{n}-x_{n}}{r_{n}}\right\rangle+F_{1}\left(z_{t}, z_{n}\right) .
\end{aligned}
$$

Since $\left\|x_{n}-z_{n}\right\| \rightarrow 0, n \rightarrow \infty$, we obtain $\left\|A x_{n}-A z_{n}\right\| \rightarrow 0, n \rightarrow \infty$. Furthermore, by the monotonicity of $A$, we obtain $\left\langle z_{t}-z_{n}, A z_{t}-A z_{n}\right\rangle \geq 0$. Then, by (A4) we obtain (noting that $z_{n} \rightarrow z, n \rightarrow \infty$ since $\left.\left\|z_{n}-z\right\| \leq\left\|x_{n}-z\right\|+\left\|x_{n}-z_{n}\right\|\right)$,

$$
\left\langle z_{t}-z, A z_{t}\right\rangle \geq \varphi_{1}(z)-\varphi_{1}\left(z_{t}\right)+F_{1}\left(z_{t}, z\right)
$$

Using (A1), (A4) and (3.44), we also obtain

$$
\begin{aligned}
0= & F_{1}\left(z_{t}, z_{t}\right)+\varphi_{1}\left(z_{t}\right)-\varphi_{1}\left(z_{t}\right) \leq t F_{1}\left(z_{t}, y\right)+(1-t) F_{1}\left(z_{t}, z\right) \\
& +t \varphi_{1}(y)+(1-t) \varphi_{1}(z)-\varphi_{1}\left(z_{t}\right) \\
\leq & t\left[F_{1}\left(z_{t}, y\right)+\varphi_{1}(y)-\varphi_{1}\left(z_{t}\right)\right]+(1-t)\left\langle z_{t}-z, A z_{t}\right\rangle \\
= & t\left[F_{1}\left(z_{t}, y\right)+\varphi_{1}(y)-\varphi_{1}\left(z_{t}\right)\right]+(1-t) t\left\langle y-z, A z_{t}\right\rangle,
\end{aligned}
$$

and hence

$$
0 \leq F_{1}\left(z_{t}, y\right)+\varphi_{1}(y)-\varphi_{1}\left(z_{t}\right)+(1-t)\left\langle y-z, A z_{t}\right\rangle
$$

Letting $t \rightarrow 0$, we have, for each $y \in K$,

$$
0 \leq F_{1}(z, y)+\varphi_{1}(y)-\varphi_{1}(z)+\langle y-z, A z\rangle
$$


This implies that $z \in \operatorname{GMEP}\left(F_{1}, A, \varphi_{1}\right)$. By following the same arguments, we can show that $z \in \operatorname{GMEP}\left(F_{2}, B, \varphi_{2}\right)$.

Next, we show $z \in V(K, D)$. Put

$$
M x= \begin{cases}D x+N_{K} x, & x \in K, \\ \emptyset, & x \notin K .\end{cases}
$$

Since $D$ is relaxed $(\lambda, \gamma)$-cocoercive and by condition (iv), we have

$$
\langle D x-D y, x-y\rangle \geq(-\lambda)\|D x-D y\|^{2}+\gamma\|x-y\|^{2} \geq\left(\gamma-\lambda \mu^{2}\right)\|x-y\|^{2} \geq 0,
$$

which shows that $D$ is monotone. Thus, $M$ is maximal monotone. Let $(x, y) \in G(M)$. Since $y-D x \in N_{K} x$ and $w_{n} \in K$, we have

$$
\left\langle x-w_{n}, y-D x\right\rangle \geq 0
$$

On the other hand, from $w_{n}=P_{\mathrm{K}}\left(I-s_{n} A\right) u_{n}$, we have

$$
\left\langle x-w_{n}, w_{n}-\left(I-s_{n} D\right) u_{n}\right\rangle \geq 0
$$

and hence

$$
\left\langle x-w_{n}, \frac{w_{n}-u_{n}}{s_{n}}+D u_{n}\right\rangle \geq 0
$$

It follows that

$$
\begin{aligned}
\left\langle x-w_{n}, y\right\rangle & \geq\left\langle x-w_{n}, D x\right\rangle \geq\left\langle x-w_{n}, y\right\rangle-\left\langle x-w_{n}, \frac{w_{n}-u_{n}}{s_{n}}+D u_{n}\right\rangle \\
& =\left\langle x-w_{n}, D x-\frac{w_{n}-u_{n}}{s_{n}}-D u_{n}\right\rangle \\
& \geq\left\langle x-w_{n}, D w_{n}-D u_{n}\right\rangle-\left\langle x-w_{n}, \frac{\rho_{n}-u_{n}}{s_{n}}\right\rangle,
\end{aligned}
$$

which implies that $\langle x-z, y\rangle \geq 0$. We have $z \in M^{-1} 0$ and hence $z \in \operatorname{VI}(K, D)$. Therefore, $z \in \cap_{i=1}^{\infty} F\left(T_{i}\right) \cap \operatorname{GMEP}\left(F_{1}, A, \varphi_{1}\right) \cap \operatorname{GMEP}\left(F_{2}, B, \varphi_{2}\right) \cap \operatorname{VI}(K, D)$.

Noting that $x_{n}=P_{C_{n}} x_{0}$, we have by (2.3) that

$$
\left\langle x_{0}-x_{n}, y-x_{n}\right\rangle \leq 0
$$


for all $y \in C_{n}$. Since $\Omega \subset C_{n}$ and by the continuity of inner product, we obtain from the above inequality that

$$
\left\langle x_{0}-z, y-z\right\rangle \leq 0
$$

for all $y \in \Omega$. By (2.3) again, we conclude that $z=P_{\Omega} x_{0}$. This completes the proof.

Corollary 3.2. Let $K$ be a nonempty closed and convex subset of a real Hilbert space H. For each $m=1,2$, let $F_{m}$ be a bifunction from $K \times K$ satisfying $(A 1)-(A 4), \varphi_{m}: K \rightarrow \mathbb{R} \cup\{+\infty\}$ a proper lower semicontinuous and convex function with assumption (B1) or (B2), A an $\alpha$-inverse-strongly monotone mapping of $K$ into $H, B$ a $\beta$-inverse-strongly monotone mapping of $K$ into $H$ and for each $i=1,2, \ldots$, let $T_{i}: K \rightarrow K$ be a nonexpansive mapping such that $\cap_{i=1}^{\infty} F\left(T_{i}\right) \neq \emptyset$. Let $D$ be a $\mu$-Lipschitzian, relaxed $(\lambda, \gamma)$-cocoercive mapping of $K$ into $H$. Suppose $\Omega:=\cap_{i=1}^{\infty} F\left(T_{i}\right) \cap \operatorname{GMEP}\left(F_{1}, A, \varphi_{1}\right) \cap$ $\operatorname{GMEP}\left(F_{2}, B, \varphi_{2}\right) \cap \mathrm{VI}(K, D) \neq \emptyset$. Let $\left\{z_{n}\right\}_{n=1}^{\infty},\left\{u_{n}\right\}_{n=1}^{\infty},\left\{w_{n}\right\}_{n=1}^{\infty},\left\{y_{n, i}\right\}_{n=1}^{\infty}(i=1,2, \ldots)$ and $\left\{x_{n}\right\}_{n=0}^{\infty}$ be generated by $x_{0} \in K, C_{1, i}=K, C_{1}=\cap_{i=1}^{\infty} C_{1, i}, x_{1}=P_{C_{1}} x_{0}$,

$$
\begin{gathered}
z_{n}=T_{r_{n}}^{\left(F_{1}, \varphi_{1}\right)}\left(x_{n}-r_{n} A x_{n}\right), \\
u_{n}=T_{\left.\lambda_{n}, \varphi_{2}\right)}^{\left(F_{2}\right)}\left(z_{n}-\lambda_{n} B z_{n}\right), \\
w_{n}=P_{K}\left(u_{n}-s_{n} D u_{n}\right), \\
y_{n, i}=\alpha_{n, i} w_{n}+\left(1-\alpha_{n, i}\right) T_{i} w_{n}, \\
C_{n+1, i}=\left\{z \in C_{n, i}:\left\|y_{n, i}-z\right\| \leq\left\|x_{n}-z\right\|\right\}, \\
C_{n+1}=\bigcap_{i=1}^{\infty} C_{n+1, i}, \\
x_{n+1}=P_{C_{n+1}} x_{0}, \quad n \geq 1 .
\end{gathered}
$$

Assume that $\left\{\alpha_{n, i}\right\}_{n=1}^{\infty} \subset[0,1)(i=1,2, \ldots),\left\{r_{n}\right\}_{n=1}^{\infty} \subset[0,2 \alpha]$, and $\left\{\lambda_{n}\right\}_{n=1}^{\infty} \subset[0,2 \beta]$ satisfy

(i) $0<a \leq r_{n} \leq b<2 \alpha$,

(ii) $0<c \leq \lambda_{n} \leq f<2 \beta$,

(iii) $0 \leq \alpha_{n, i} \leq d_{i}<1$,

(iv) $0<h \leq s_{n} \leq j<2\left(\gamma-\lambda \mu^{2}\right) / \mu^{2}$.

Then, $\left\{x_{n}\right\}_{n=0}^{\infty}$ converges strongly to $P_{\Omega} x_{0}$.

Let $C$ be a nonempty closed and convex cone in $H$ and $D$ an operator of $C$ into $H$. We define the polar of $C$ in $H$ to be the set

$$
K^{*}:=\left\{y^{*} \in H:\left\langle x, y^{*}\right\rangle \geq 0, \forall x \in C\right\}
$$

Then, the element $u \in C$ is called a solution of the complementarity problem if

$$
D u \in K^{*}, \quad\langle u, D u\rangle=0 .
$$


The set of solutions of the complementarity problem is denoted by $C(C, D)$. We shall assume that $D$ satisfies the following conditions:

(E1) $D$ is $\gamma$-inverse strongly monotone,

(E2) $C(C, D) \neq \emptyset$.

Also, we replace conditions (B1) and (B2) with

(D1) for each $x \in H$ and $r>0$ there exist a bounded subset $D_{x} \subseteq C$ and $y_{x} \in C$ such that for any $z \in C \backslash D_{x}$,

$$
F\left(z, y_{x}\right)+\varphi\left(y_{x}\right)-\varphi(z)+\frac{1}{r}\left\langle y_{x}-z, z-x\right\rangle<0,
$$

(D2) $C$ is a bounded set.

Theorem 3.3. Let $C$ be a nonempty closed and convex cone of a real Hilbert space $H$. For each $m=1,2$, let $F_{m}$ be a bifunction from $C \times C$ satisfying (A1)-(A4), $\varphi_{m}: C \rightarrow \mathbb{R} \cup\{+\infty\}$ a proper lower semicontinuous and convex function with assumption (D1) or (D2), $A$ an $\alpha$-inversestrongly monotone mapping of $C$ into $H, B$ a $\beta$-inverse-strongly monotone mapping of $C$ into $H$ and for each $i=1,2, \ldots$, let $T_{i}: C \rightarrow C$ be a $k_{i}$-strictly pseudocontractive mapping for some $0 \leq k_{i}<1$ such that $\cap_{i=1}^{\infty} F\left(T_{i}\right) \neq \emptyset$. Let $D$ be a $\mu$-Lipschitzian, relaxed $(\lambda, \gamma)$-cocoercive mapping of $C$ into $H$. Suppose $\Omega:=\cap_{i=1}^{\infty} F\left(T_{i}\right) \cap \operatorname{GMEP}\left(F_{1}, A, \varphi_{1}\right) \cap \operatorname{GMEP}\left(F_{2}, B, \varphi_{2}\right) \cap C(C, D) \neq \emptyset$. Let $\left\{z_{n}\right\}_{n=1}^{\infty},\left\{u_{n}\right\}_{n=1}^{\infty},\left\{w_{n}\right\}_{n=1}^{\infty},\left\{y_{n, i}\right\}_{n=1}^{\infty}(i=1,2, \ldots)$, and $\left\{x_{n}\right\}_{n=0}^{\infty}$ be generated by $x_{0} \in C, C_{1, i}=$ $C, C_{1}=\cap_{i=1}^{\infty} C_{1, i}, x_{1}=P_{C_{1}} x_{0}$,

$$
\begin{aligned}
& z_{n}=T_{r_{n}}^{\left(F_{1}, \varphi_{1}\right)}\left(x_{n}-r_{n} A x_{n}\right), \\
& u_{n}=T_{\lambda_{n}}^{\left(F_{2}, \varphi_{2}\right)}\left(z_{n}-\lambda_{n} B z_{n}\right), \\
& w_{n}=P_{C}\left(u_{n}-s_{n} D u_{n}\right), \\
& y_{n, i}=\alpha_{n, i} w_{n}+\left(1-\alpha_{n, i}\right) T_{i} w_{n} \\
& C_{n+1, i}=\left\{z \in C_{n, i}:\left\|y_{n, i}-z\right\| \leq\left\|x_{n}-z\right\|\right\}, \\
& C_{n+1}=\bigcap_{i=1}^{\infty} C_{n+1, i} \\
& x_{n+1}=P_{C_{n+1}} x_{0}, \quad n \geq 1 .
\end{aligned}
$$

Assume that $\left\{\alpha_{n, i}\right\}_{n=1}^{\infty} \subset[0,1)(i=1,2, \ldots),\left\{r_{n}\right\}_{n=1}^{\infty} \subset[0,2 \alpha]$, and $\left\{\lambda_{n}\right\}_{n=1}^{\infty} \subset[0,2 \beta]$ satisfy

(i) $0<a \leq r_{n} \leq b<2 \alpha$ ，

(ii) $0<c \leq \lambda_{n} \leq f<2 \beta$,

(iii) $0 \leq k_{i} \leq \alpha_{n, i} \leq d_{i}<1$,

(iv) $0<h \leq s_{n} \leq j<2\left(\gamma-\lambda \mu^{2}\right) / \mu^{2}$.

Then, $\left\{x_{n}\right\}_{n=0}^{\infty}$ converges strongly to $P_{\Omega} x_{0}$. 
Proof. Using Lemma 7.1.1 of [34], we have that $\operatorname{VI}(C, D)=C(C, D)$. Hence, by Theorem 3.1, we obtain the desired conclusion.

Remark 3.4. Our Corollary 3.2 extends Theorems 1.1 and 1.2.

Remark 3.5. Our iterative scheme (3.1) is simpler than the iterative schemes (5.1) and (5.11) of Acedo and $\mathrm{Xu}$ [6]. Furthermore, in our results, we use iterative scheme (3.1) to approximate a common fixed point of an infinite family of $k$-strictly pseudocontractive mappings while the iterative schemes (5.1) and (5.11) of Acedo and Xu [6] are used to approximate a common fixed point of a finite family of $k$-strictly pseudocontractive mappings.

Remark 3.6. Our results also hold for infinite family of uniformly continuous quasistrict pseudocontractions. Hence, we can adapt our results for an infinite family of uniformly continuous quasi-nonexpansive mappings in a real Hilbert space.

\section{Acknowledgments}

The author is extremely grateful to Professor S. Al-Homidan and the anonymous referees for their valuable comments and useful suggestions which improve the presentation of this paper. This research work is dedicated to Professor C. E. Chidume with admiration and respect.

\section{References}

[1] F. E. Browder and W. V. Petryshyn, "Construction of fixed points of nonlinear mappings in Hilbert space," Journal of Mathematical Analysis and Applications, vol. 20, pp. 197-228, 1967.

[2] H. Iiduka and W. Takahashi, "Strong convergence theorems for nonexpansive mappings and inversestrongly monotone mappings," Nonlinear Analysis: Theory, Methods E Applications, vol. 61, no. 3, pp. 341-350, 2005.

[3] R. E. Bruck Jr., "On the weak convergence of an ergodic iteration for the solution of variational inequalities for monotone operators in Hilbert space," Journal of Mathematical Analysis and Applications, vol. 61, no. 1, pp. 159-164, 1977.

[4] L.-C. Ceng, A. R. Khan, Q. H. Ansari, and J.-C. Yao, "Viscosity approximation methods for strongly positive and monotone operators," Fixed Point Theory, vol. 10, no. 1, pp. 35-72, 2009.

[5] R. T. Rockafellar, "Monotone operators and the proximal point algorithm," SIAM Journal on Control and Optimization, vol. 14, no. 5, pp. 877-898, 1976.

[6] G. L. Acedo and H.-K. Xu, "Iterative methods for strict pseudo-contractions in Hilbert spaces," Nonlinear Analysis: Theory, Methods E Applications, vol. 67, no. 7, pp. 2258-2271, 2007.

[7] Y. J. Cho, X. Qin, and J. I. Kang, "Convergence theorems based on hybrid methods for generalized equilibrium problems and fixed point problems," Nonlinear Analysis: Theory, Methods E Applications, vol. 71, no. 9, pp. 4203-4214, 2009.

[8] Y. Liu, "A general iterative method for equilibrium problems and strict pseudo-contractions in Hilbert spaces," Nonlinear Analysis: Theory, Methods E Applications, vol. 71, no. 10, pp. 4852-4861, 2009.

[9] H. Zhou, "Convergence theorems of fixed points for $\kappa$-strict pseudo-contractions in Hilbert spaces," Nonlinear Analysis: Theory, Methods E Applications, vol. 69, no. 2, pp. 456-462, 2008.

[10] M. Liu, S. Chang, and P. Zuo, "On a hybrid method for generalized mixed equilibrium problem and fixed point problem of a family of quasi- $\phi$-asymptotically nonexpansive mappings in Banach spaces," Fixed Point Theory and Applications, vol. 2010, Article ID 157278, 18 pages, 2010.

[11] N. Petrot, K. Wattanawitoon, and P. Kumam, "A hybrid projection method for generalized mixed equilibrium problems and fixed point problems in Banach spaces," Nonlinear Analysis: Hybrid Systems, vol. 4, no. 4, pp. 631-643, 2010. 
[12] S. Zhang, "Generalized mixed equilibrium problem in Banach spaces," Applied Mathematics and Mechanics. English Edition, vol. 30, no. 9, pp. 1105-1112, 2009.

[13] P. L. Combettes and S. A. Hirstoaga, "Equilibrium programming in Hilbert spaces," Journal of Nonlinear and Convex Analysis, vol. 6, no. 1, pp. 117-136, 2005.

[14] S. Plubtieng and R. Punpaeng, "A new iterative method for equilibrium problems and fixed point problems of nonexpansive mappings and monotone mappings," Applied Mathematics and Computation, vol. 197, no. 2, pp. 548-558, 2008.

[15] X. Qin, M. Shang, and Y. Su, "Strong convergence of a general iterative algorithm for equilibrium problems and variational inequality problems," Mathematical and Computer Modelling, vol. 48, no. 7-8, pp. 1033-1046, 2008.

[16] Y. Su, M. Shang, and X. Qin, "An iterative method of solution for equilibrium and optimization problems," Nonlinear Analysis: Theory, Methods \& Applications, vol. 69, no. 8, pp. 2709-2719, 2008.

[17] S. Takahashi and W. Takahashi, "Viscosity approximation methods for equilibrium problems and fixed point problems in Hilbert spaces," Journal of Mathematical Analysis and Applications, vol. 331, no. 1, pp. 506-518, 2007.

[18] X. Qin, Y. J. Cho, and S. M. Kang, "Viscosity approximation methods for generalized equilibrium problems and fixed point problems with applications," Nonlinear Analysis: Theory, Methods $\mathcal{E}$ Applications, vol. 72, no. 1, pp. 99-112, 2010.

[19] Y. Shehu, "Fixed point solutions of generalized equilibrium problems for nonexpansive mappings," Journal of Computational and Applied Mathematics, vol. 234, no. 3, pp. 892-898, 2010.

[20] S. Takahashi and W. Takahashi, "Strong convergence theorem for a generalized equilibrium problem and a nonexpansive mapping in a Hilbert space," Nonlinear Analysis: Theory, Methods E Applications, vol. 69, no. 3, pp. 1025-1033, 2008.

[21] J.-W. Peng and J.-C. Yao, "Strong convergence theorems of iterative scheme based on the extragradient method for mixed equilibrium problems and fixed point problems," Mathematical and Computer Modelling, vol. 49, no. 9-10, pp. 1816-1828, 2009.

[22] S. Plubtieng and K. Sombut, "Weak convergence theorems for a system of mixed equilibrium problems and nonspreading mappings in a Hilbert space," Journal of Inequalities and Applications, vol. 2010, Article ID 246237, 12 pages, 2010.

[23] Y. Yao, Y.-C. Liou, and J.-C. Yao, "A new hybrid iterative algorithm for fixed-point problems, variational inequality problems, and mixed equilibrium problems," Fixed Point Theory and Applications, vol. 2008, Article ID 417089, 15 pages, 2008.

[24] E. Blum and W. Oettli, "From optimization and variational inequalities to equilibrium problems," The Mathematics Student, vol. 63, no. 1-4, pp. 123-145, 1994.

[25] L.-C. Ceng and J.-C. Yao, "A hybrid iterative scheme for mixed equilibrium problems and fixed point problems," Journal of Computational and Applied Mathematics, vol. 214, no. 1, pp. 186-201, 2008.

[26] N. Nadezhkina and W. Takahashi, "Weak convergence theorem by an extragradient method for nonexpansive mappings and monotone mappings," Journal of Optimization Theory and Applications, vol. 128, no. 1, pp. 191-201, 2006.

[27] M. A. Noor, "General variational inequalities and nonexpansive mappings," Journal of Mathematical Analysis and Applications, vol. 331, no. 2, pp. 810-822, 2007.

[28] P. Kumam, "A new hybrid iterative method for solution of equilibrium problems and fixed point problems for an inverse strongly monotone operator and a nonexpansive mapping," Journal of Applied Mathematics and Computing, vol. 29, no. 1-2, pp. 263-280, 2009.

[29] X. Qin, Y. J. Cho, and S. M. Kang, "Convergence theorems of common elements for equilibrium problems and fixed point problems in Banach spaces," Journal of Computational and Applied Mathematics, vol. 225, no. 1, pp. 20-30, 2009.

[30] R. Wangkeeree and R. Wangkeeree, "A general iterative method for variational inequality problems, mixed equilibrium problems, and fixed point problems of strictly pseudocontractive mappings in Hilbert spaces," Fixed Point Theory and Applications, vol. 2009, Article ID 519065, 32 pages, 2009.

[31] M. Liu, S. S. Chang, and P. Zuo, "An algorithm for finding a common solution for a system of mixed equilibrium problem, quasi-variational inclusion problem, and fixed point problem of nonexpansive semigroup," Journal of Inequalities and Applications, vol. 2010, Article ID 895907, 23 pages, 2010.

[32] Y. Yao, M. A. Noor, S. Zainab, and Y.-C. Liou, "Mixed equilibrium problems and optimization problems," Journal of Mathematical Analysis and Applications, vol. 354, no. 1, pp. 319-329, 2009. 
[33] W. Takahashi, Y. Takeuchi, and R. Kubota, "Strong convergence theorems by hybrid methods for families of nonexpansive mappings in Hilbert spaces," Journal of Mathematical Analysis and Applications, vol. 341, no. 1, pp. 276-286, 2008.

[34] W. Takahashi, Nonlinear Functional Analysis, Fixed Point Theory and Its Application, Yokohama Publishers, Yokohama, Japan, 2000. 